\title{
Reducing signaling load for power-efficient integrated WLAN/cellular networks
}

\author{
Yun Won Chung ${ }^{\text {a) }}$ \\ School of Electronic Engineering, Soongsil University \\ 511 Sangdo-dong, Dongjak-gu, Seoul, 156-743, Korea \\ a)ywchung@ssu.ac.kr
}

\begin{abstract}
In power-efficient WLAN/cellular dual mode mobile station (MS), WLAN interface is basically turned off when it is not actively transmitting or receiving data because idle state power consumption of WLAN interface is significant and it is much higher than that of cellular interface. Since only cellular interface is turned on to maintain reachability, if a VoIP call is arrived at WLAN it is notified through less power-consuming cellular interface. The paging via cellular interface for VoIP call, however, generates significant signaling load at radio interface because paging has to be performed to all cells within a registered cellular registration area (RA). In this paper, we reduce signaling load of power-efficient WLAN/cellular networks by paging cells only containing WLAN using the network coverage information between WLAN and cellular network, without degrading the performance of power consumption. Numerical results show that the signaling load of power-efficient integrated WLAN/cellular networks is significantly reduced.
\end{abstract}

Keywords: integrated WLAN/cellular networks, dual mode mobile station, paging, signaling load, power consumption

Classification: Science and engineering for electronics

\section{References}

[1] Y. Agarwal, R. Chandra, A. Wolman, P. Bahl, K. Chin, and R. Gupta, "Wireless wakeups revisited: energy management for VoIP over Wi-Fi smartphones," in Proc. IEEE MobiSys'07, June 2007.

[2] S. H. Tsao and E. C. Cheng, "PIANO: a power saving strategy for cellular/VoWLAN dual-mode mobiles," Wireless Networks, vol. 14, no. 5, pp. 683-698, Oct. 2008.

[3] S. K. Lee, W. S. Chung, K. H. Hong, and N. Golmie, "Power-efficient communication protocol for integrated WWAN and WLAN," J. Wireless Commun. Netw., vol. 2008, pp. 1-13, 2008.

[4] S. H. Seo and J. S. Song, "An energy-efficient interface selection for multimode terminals by utilizing out-of-band paging channels," Telecommunication Syst., vol. 42, no. 1-2, pp. 151-161, Oct. 2009.

[5] G. Lampropoulos, N. Passas, L. Merakos, and A. Kaloxylos, "Handover management architectures in integrated WLAN/cellular networks," IEEE Commun. Surveys, vol. 7, no. 4, pp. 30-44, 2005. 
[6] I. F. Akyildiz, J. S. Ho, and Y. B. Lin, "Movement-based location update and selective paging for PCS networks," IEEE/ACM Trans. Netw., vol. 4, pp. 629-638, Aug. 1996.

[7] Y. Watanabe and M. Yabusaki, "Mobility/traffic adaptive location management," IEICE Trans. Commun., vol. E85-B, no. 10, pp. 2076-2082, 2002.

\section{Introduction}

Various wireless access networks, such as cellular network, wireless local area network (WLAN), Mobile WiMAX, and satellite network are complementary to each other in terms of coverage, mobility, bandwidth, etc. For example, cellular network provides high mobility with large coverage area. On the other hand, WLAN provides low mobility but high bandwidth with cheap price. Thus, mobile users can receive efficient services by selecting an appropriate access network with multi radio mobile stations (MSs). Out of these access networks, we focus on WLAN and cellular networks, i.e., IEEE 802.11-based WLAN and 3GPP GPRS/UMTS, in this paper, because they are widely deployed and dual mode MSs with WLAN/cellular networks interfaces are already available in the market.

With dual mode MS with WLAN/cellular networks interfaces, mobile users can enjoy cheap voice over IP (VoIP) service over WLAN and high mobility service through cellular network. In dual mode MS, however, battery power consumption is significant since multiple radio interfaces operate simultaneously. In order to solve the power consumption problem of dual mode MS, works on efficient power consumption in WLAN/celluar dual mode MS have been studied actively $[1,2,3,4]$. In these works, WLAN interface is basically turned off when it is not actively transmitting or receiving data, and WLAN interface only wakes up to transmit or receive data. This is because idle state power consumption of WLAN interface is significant and it is much higher than that of cellular interface. On the other hand, cellular interface is always turned on to maintain reachability. Therefore, if a VoIP call is arrived at WLAN, it is notified through less power-consuming cellular interface. Since the location of MSs in cellular network is tracked by registration area (RA) consisting of a set of cells, paging requests are sent to all cells within the registered RA through cellular interface. Then, the dual mode MS wakes up its WLAN interface and VoIP call is delivered via WLAN interface.

The paging via cellular interface for VoIP call, however, generates significant signaling load at radio interface and wastes scarce radio resources, because paging has to be performed to all cells within a registered cellular RA, although WLAN may exist only in part of cells within a cellular RA. In this paper, we reduce signaling load of power-efficient WLAN/cellular networks by paging cells only containing WLAN using the network coverage information between WLAN and cellular network, without degrading the 
performance of power consumption.

\section{Proposed signaling reduction scheme}

Fig. 1 shows a simple interworked WLAN and cellular network architecture, where session initiation protocol (SIP) server is located within Internet. WLAN and cellular network can be interworked, either based on tight coupling or loose coupling [5]. It is assumed that WLAN is managed by wireless Internet service providers (WISPs) for efficient interworking between WLAN and cellular networks. Since the service coverage of WLAN hotspots of WISPs does not cover all the cellular network area yet, WLAN only exists in part of cells within an RA. It is expected that the ratio of cells containing WLAN within an RA is high in downtown but it is low in rural area.

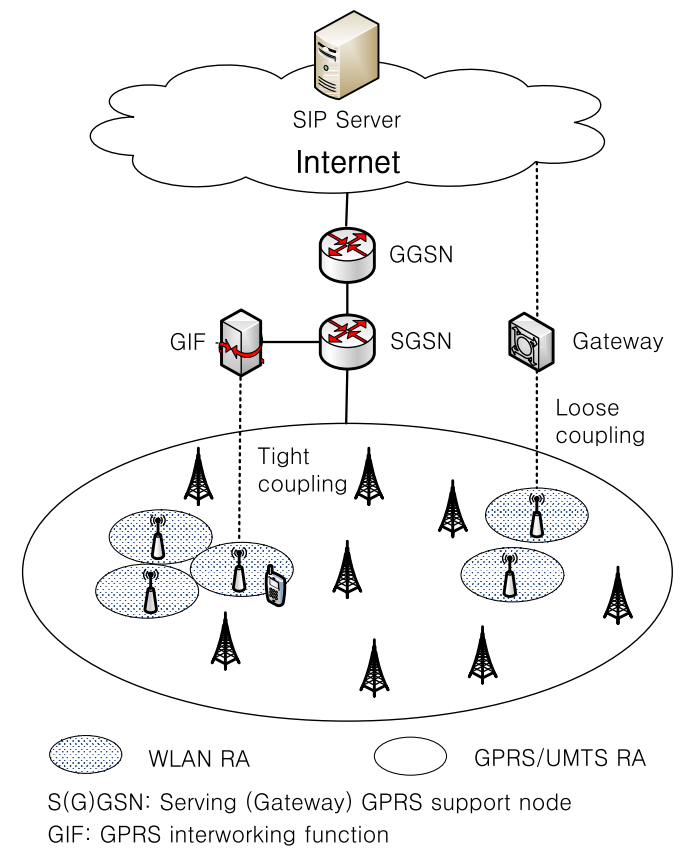

Fig. 1. Interworked WLAN and cellular network.

If an incoming VoIP call is arrived at SIP server, it sends a call request to cellular network since the location of dual mode MS is tracked by the cellular network. Then, cellular network pages the called MS through cellular interface since WLAN interface is turned off. In works in $[1,2,3,4]$, denoted by conventional scheme in this paper for notational convenience, paging requests are sent to all cells within the registered cellular RA. On the other hand, paging requests are sent only to cells containing WLAN within a registered RA in the proposed scheme. Upon receiving the paging message via cellular interface, the called MS wakes up its WLAN interface. Then, it responds to the SIP server via WLAN interface by sending SIP register message. Then, SIP invite message is delivered from SIP server to the called MS via the WLAN interface and the VoIP call is successfully delivered after ringing and acknowledgment procedures. 
The proposed scheme can be realized in integrated WLAN/cellular networks practically because cellular network can obtain the location information of WLAN coverage via online or offline. For example, virtual map of APs (VMAP) [4], which is hierarchically located in Node B and Serving GPRS support node (SGSN), can manage a geographical location mapping information between APs' and Node B's coverage area because each AP updates its location information to the VMAP whenever it initializes its operation. Based on this information, cellular network can obtain the cell identities having WLAN within an RA. Also, cellular network operator can get the location information of installed APs from interworked WISPs via offline and use this information to obtain cell identities having WLAN.

If the called MS is out of WLAN coverage in the conventional scheme, the MS cannot respond to the SIP server via WLAN interface. In this case, the MS responds to the paging via cellular interface after the expiration of WLAN connection setup request and the VoIP call is delivered via cellular network. In the proposed scheme, since the initial paging is only performed to the cells containing WLAN, the called MS does not receive any paging message, if it is located out of WLAN coverage. Thus, if cellular network does not receive any response until paging timer expires, it sends an additional paging requests to the remaining cells within the registered RA, and VoIP call is successfully delivered via cellular network, too. We note that the proposed scheme does not degrade the performance of power consumption of dual mode MS, compared with conventional scheme, since there is little difference in MS power state managements between the two schemes.

\section{Performance analysis and numerical examples}

In this section, the performance of the proposed scheme is compared with that of the conventional scheme in terms of signaling load at radio interface. Without loss of generality, we assume the followings for performance analysis:

- VoIP call arrival follows a Poisson process with parameter $\lambda_{c}$.

- Cell and RA residence times of dual mode MS in cellular network follow an exponential distribution with parameters $\mu_{\text {cell }}$ and $\mu_{R A}$, respectively.

- The number of cells within an RA is $N_{\text {cell }}$.

- The ratio of cells containing WLAN within an RA is $P_{W}$.

- The probability that an MS is located within WLAN coverage when a VoIP call arrives is $P_{h i t}$

Based on the above assumptions, the signaling load at radio interface per unit time is obtained as the weighted sum of location registration and paging signaling load as follows:

$$
\begin{aligned}
S_{\text {conv }} & =w_{\text {reg }} \mu_{R A}+w_{\text {pag }} \lambda_{c} N_{\text {cell }} . \\
S_{\text {prop }} & =w_{\text {reg }} \mu_{R A}+w_{\text {pag }} \lambda_{c}\left(P_{W} N_{\text {cell }} P_{\text {hit }}+N_{\text {cell }}\left(1-P_{\text {hit }}\right)\right) \\
& =w_{\text {reg }} \mu_{R A}+w_{\text {pag }} \lambda_{c} N_{\text {cell }}\left(1-P_{\text {hit }}\left(1-P_{W}\right)\right) .
\end{aligned}
$$


In Eq. (1) and Eq. (2), $w_{r e g}$ and $w_{p a g}$ are weight factors of location registration and paging signaling load [6]. In Eq. (1), paging signaling load is irrelevant to $P_{W}$ in the conventional scheme since paging is performed to all cells within registered RA. In the proposed scheme, however, $N_{\text {cell }} P_{W}$ cells are searched first in the initial paging request and the called MS responds to the paging request with the probability of $P_{\text {hit }}$. If the called MS is located out of WLAN coverage with the probability of $1-P_{h i t}$ and does not respond to the initial paging request, additional paging is performed to the remaining cells.

Since the objective of this paper is to compare the relative performance of the conventional and proposed schemes, the shape of cells and RAs are assumed as square for mathematical simplicity. Also, fluid flow mobility model [7] is assumed as mobility model for an MS. Based on the square shape of cell and RA and fluid flow mobility model, the following relationship [7] holds:

$$
\mu_{R A}=\mu_{\text {cell }} / \sqrt{N_{\text {cell }}} \text {. }
$$

Applying the above relationship to Eq. (1) and Eq. (2) yields the following equations:

$$
\begin{aligned}
& S_{\text {conv }}=w_{\text {reg }} \mu_{\text {cell }} / \sqrt{N_{\text {cell }}}+w_{\text {pag }} \lambda_{c} N_{\text {cell }} . \\
& S_{\text {prop }}=w_{\text {reg }} \mu_{\text {cell }} / \sqrt{N_{\text {cell }}}+w_{\text {pag }} \lambda_{c} N_{\text {cell }}\left(1-P_{\text {hit }}\left(1-P_{W}\right)\right) .
\end{aligned}
$$

Fig. 2 (a) shows signaling load for varying the values of $P_{W}$, with $w_{r e g}=$ 10 [6], $w_{\text {pag }}=1[6], N_{\text {cell }}=36, \mu_{\text {cell }}=10(/ h)$, and $\lambda_{c}=3.0(/ h)$. As expected, the proposed scheme performs better than the conventional scheme, especially for low values of $P_{W}$ and high values of $P_{\text {hit }}$. For example, the signaling load of the proposed scheme for $P_{W}=0.2$ and $P_{h i t}=0.6$ is only about $60 \%$ of that of the conventional scheme.

Fig. 2 (b) shows signaling load for varying the values of $P_{W}$, with the same parameter values, as in Fig. 2 (a), with $P_{h i t}=P_{W}$ by assuming uniform distribution of MSs in service area. Although the proposed scheme has the same signaling load for two extreme cases, i.e., $P_{W}=0$ (there is no cell containing WLAN) or $P_{W}=1$ (all the cells contain WLAN), it always performs better than the conventional scheme, otherwise. Especially, it achieves the largest signaling load reduction of about $20 \%$ for $P_{W}=0.5$.

Fig. 2 (c) shows signaling load for varying the values of $N_{\text {cell }}$, with $w_{\text {reg }}=$ $10[6], w_{\text {pag }}=1[6], \mu_{\text {cell }}=10(/ h), \lambda_{c}=3.0(/ h)$, and $P_{W}=0.5$. The proposed scheme performs better than the conventional scheme, especially for high values of $N_{\text {cell }}$ and $P_{h i t}$. Given value of $P_{h i t}$, the performance enhancement is more significant for large values of $N_{\text {cell }}$. This is because paging signaling load has a more dominant effect on the signaling load for large values of $N_{\text {cell }}$ and the proposed scheme has better performance in terms of paging signaling load. For small values of $N_{\text {cell }}$, the signaling load decreases as the value of $N_{\text {cell }}$ increases. On the other hand, it increases as the value of $N_{\text {cell }}$ increases for large values of $N_{\text {cell }}$. This is because registration signaling 


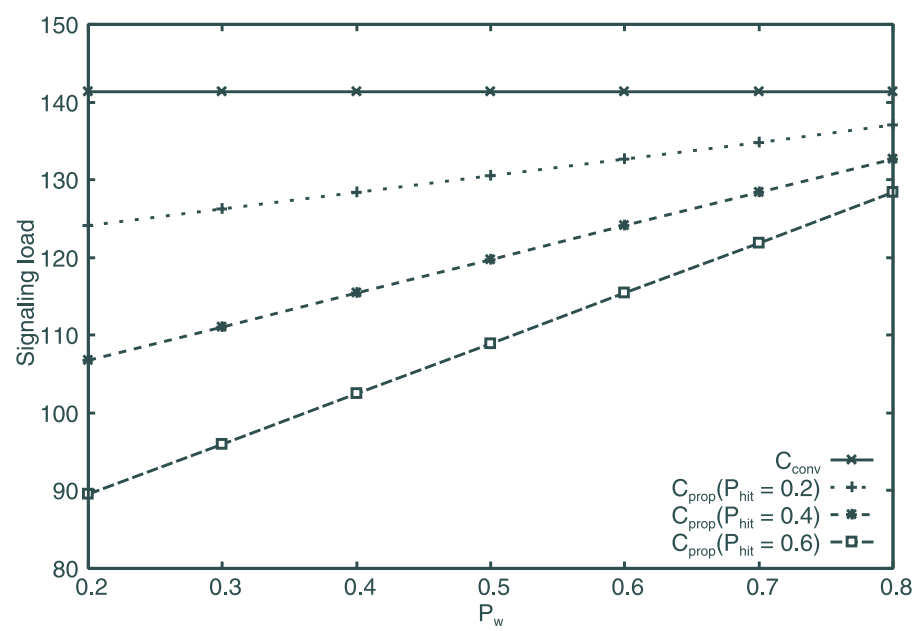

(a)

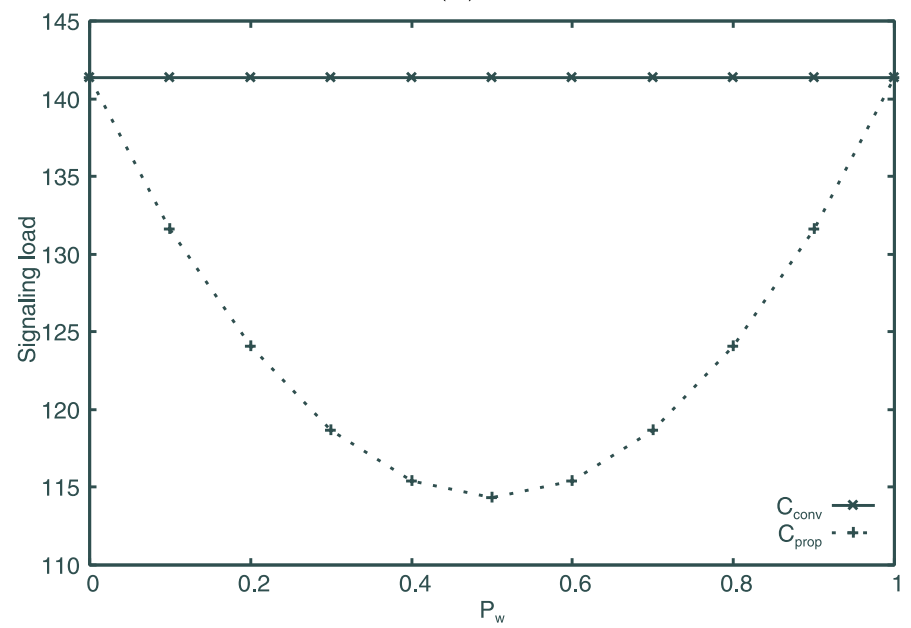

(b)

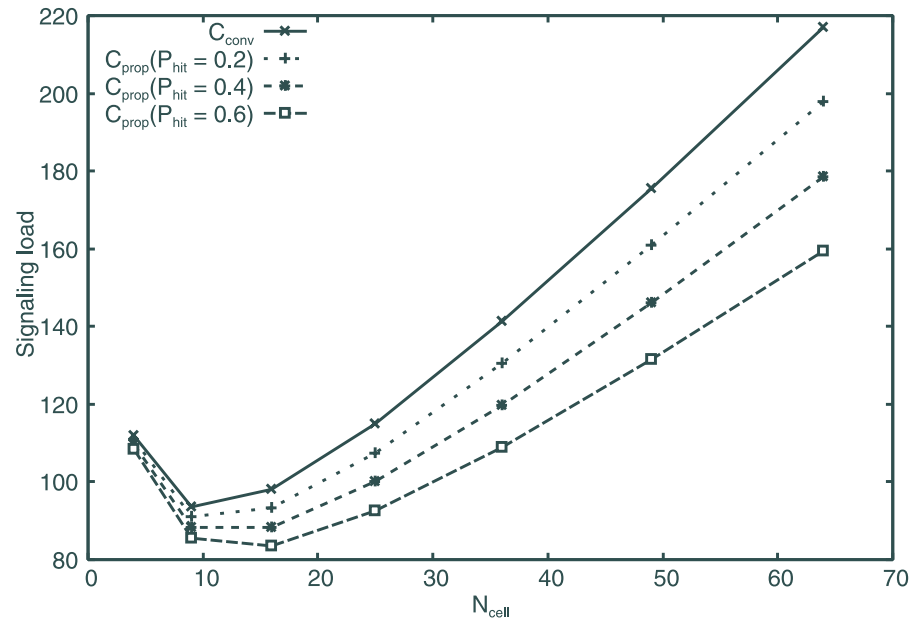

(c)

Fig. 2. Signaling load analysis for (a) varying the ratio of cells containing WLAN within an RA. (b) varying the ratio of cells containing WLAN within an RA for uniform distribution of MSs (c) varying the number of cells within an RA. 
load has a more dominant effect for small values of $N_{\text {cell }}$ and paging signaling load has a more dominant effect for large values of $N_{\text {cell }}$.

\section{Conclusion}

In this paper, we propose to page cells only containing WLAN within a registered cellular RA for VoIP call delivery, in order to reduce significant signaling load due to paging via cellular interface in power-efficient WLAN/cellular networks. From numerical examples, it can be concluded that signaling load of the conventional power-efficient WLAN/cellular networks can be reduced significantly by employing the proposed scheme, without degrading the performance of power consumption. The proposed scheme can be implemented in current cellular network practically by managing the network coverage information between WLAN and cellular networks.

\section{Acknowledgments}

This research is supported by the ubiquitous Computing and Network (UCN) Project, the Ministry of Knowledge and Economy (MKE) Knowledge and Economy Frontier R\&D Program in Korea as a result of UCN's subproject 10C2-C1-20S. 